\title{
TUMOR DE FRANTZ: EL TUMOR DE LAS MUJERES JOVENES. CORRELACION RADIOLOGICO-PATOLOGICA DE DOS CASOS EN TOMOGRAFIA REVISION DE LA LITERATURA.
}

\author{
ALVARO TAFUR ANZOLA MD"1, DANIEL SUAREZ JIMENEZ MD²" \\ ${ }^{1}$ Radiólogo. Hospital Militar Central. Docente de Radiología e Imágenes Diagnósticas. \\ Hospital Militar Central. Bogotá. Colombia. \\ ${ }^{2}$ Residente de Radiología e Imágenes Diagnósticas. Universidad Militar Nueva Granada Bogotá. Colombia \\ *Correspondencia: Daniel Suárez Jiménez dasua07@hotmail.com
}

Recibido: 6 Julio 2016 Aceptado: 3 Febrero 2017

\begin{abstract}
RESUMEN
El tumor de Frantz o tumor sólido pseudopapilar del páncreas, es un tumor raro único de bajo potencial maligno que afecta principalmente a mujeres jóvenes con un promedio de edad de 21,97 años. Las pacientes son generalmente asintomáticas y es descubierto de manera incidental mediante examen físico y la realización de imágenes por otras razones. La tasa de curación con la resección total del tumor es mayor al 95\%. Por lo tanto, el análisis realizado por las imágenes en resonancia magnética o en tomografía y la posterior confirmación patológica, demuestra con precisión las características de este tumor para su mayor comprensión y poder realizar un rápido diagnóstico y tratamiento oportuno.
\end{abstract}

Palabras clave: Tumor; páncreas; mujeres; tomografía. 


\title{
FRANTZ'S TUMOR. THE TUMOR OF YOUNG WOMEN. PATHOLOGICAL-RADIOLOGICAL CORRELATION OF TWO CASES ON TOMOGRAPHY REVIEW OF THE LITERATURE.
}

\begin{abstract}
Frantz's tumor or solid pseudopapillary tumor of the pancreas is a rare unique tumor of low potential for malignancy that mainly affects young women with a mean age of 21.97 years. Patients are generally asymptomatic and the tumor might be discovered at physical examination and the performance or abdominal imaging for other reasons. More than $95 \%$ of patients are cured by complete resection. Therefore, the analysis done by magnetic resonance imaging and tomography and the pathological confirmation shows with accuracy the main characteristics of this tumor for its better understanding and make a prompt diagnosis and pertinent treatment.
\end{abstract}

Key words: Tumor; Pancreas; Women, Tomography.

\section{TUMOR DE FRANTZ. O TUMOR DAS MULHERES JOVENS. CORRELAÇÃO PATOLÓGICA-RADIOLÓGICA DE DOIS CASOS NA TOMOGRAFIA REVISÃO DA LITERATURA.}

\begin{abstract}
RESUMO
O tumor de Frantz ou o tumor pseudopapilar sólido do pâncreas é um tumor único e raro de baixo potencial de malignidade que afeta principalmente mulheres jovens com idade média de 21,97 anos. Os pacientes geralmente são assintomáticos e o tumor pode ser descoberto no exame físico e no desempenho ou imagem abdominal por outros motivos. Mais de 95\% dos pacientes são urados por ressecção completa. Assim sendo, a análise feita por ressonância magnética e tomografia e a confirmação patológica mostram com precisão as principais características deste tumor para melhor compreensão e um diagnóstico imediato e tratamento pertinente.
\end{abstract}

Palavras-chave: Tumor; pâncreas; mulheres; tomografia. 


\section{Introducción}

El tumor de Frantz o tumor pseudopapilar sólido del páncreas fue descrito por primera vez en 1959 por Virginia Frantz. Posteriormente en 1996, la organización mundial de la salud lo denomino tumor solido pseudopapilar como un tumor distinto del páncreas exocrino. La presentación clínica no es muy clara ya que las pacientes, quienes son generalmente mujeres jóvenes en la segunda y tercera década de la vida, refieren sensación de masa abdominal de crecimiento gradual o leve dolor abdominal. Sin embargo, puede ser descubierto de manera incidental mediante el examen físico o la realización de imágenes diagnosticas por otros motivos. Las características imagenológicas en ultrasonido, en imagen por resonancia magnética y en tomografía tienen un común denominador que es una lesión encapsulada la cual realza en las diferentes modalidades de imagen. Estudios han demostrado que la tomografía y la resonancia magnética son precisas en un $60 \%$ en determinar el correcto diagnostico histológico de las lesiones quísticas del páncreas en especial el tumor solido pseudopapilar y lo más importante ayudan a diferenciar las lesiones agresivas de las no agresivas en un $75 \%$ a $90 \%$ de los casos. La tomografía computada puede ser comparable y algunas veces complementaria a las imágenes por resonancia magnética para la caracterización de estas lesiones. Por lo tanto, es de vital importancia para el radiólogo y el clínico en especial los gastroenterólogos y los cirujanos familiarizarse con este tipo de imágenes y con el algoritmo de tratamiento de este tumor para realizar así un diagnóstico temprano y preciso de esta entidad.

\section{Presentación clínica}

El tumor de Frantz o tumor solido pseudopapilar del páncreas es comúnmente diagnosticado en niñas adolescentes y mujeres jóvenes. El rango de edad es de 2 a 85 años con un promedio de edad de 21.97 años. Este tumor ha sido reportado predominantemente mujeres jóvenes de raza negra o de Asia oriental (1-5). No existe un factor hormonal o genético que explique la fuerte predilección por las mujeres jóvenes, aunque se sugiere que el tumor presenta receptores para progesterona y que puede aumentar su tasa de crecimiento con el embarazo $(6,7)$. Por lo tanto, se ha postulado que su patogénesis puede ser de origen endocrino. Otros autores afirman que el tumor crece de células acinares o células pluripotenciales $(3,5,8,9)$.
Las pacientes son generalmente asintomáticas. Sin embargo, algunas mujeres presentan síntomas inespecíficos como molestia abdominal o dolor leve con crecimiento gradual de la circunferencia abdominal $(6,10)$. Al examen físico el abdomen usualmente no es doloroso a la palpación, aunque puede haber sintomas obstructivos si el tumor crece lo suficiente para comprimir vísceras adyacentes (6). La ictericia es rara aun en pacientes con lesiones que comprometan la cabeza del páncreas. Ocasionalmente, el tumor de Frantz es descubierto como hallazgo incidental al realizar estudios imagenológicos del abdomen, en el examen físico por trauma abdominal o laparotomía realizada por otras razones $(11,12)$. Generalmente no hay laboratorios con valores anormales que llevan al diagnóstico de este tumor (niveles de amilasa sérica) ni marcadores tumorales pancreáticos (CA 19-9, antígeno carcinoembrionario, alfa-fetoproteina) (6).

\section{Características Patológicas}

El tumor sólido pseudopapilar del páncreas es un tumor de crecimiento lento. Usualmente mide entre 6.08 y $10.5 \mathrm{~cm}$. Se caracteriza por ser un tumor grande circunscrito y rodeado por una capsula con marcados cambios degenerativos por hemorragia, necrosis y cambios quísticos. Se localiza en cualquier parte del páncreas, pero es más comúnmente encontrado en la cabeza y la cola. Al análisis microscópico las células adquieren dos tipos de disposición: sólido y papilar. El componente sólido está compuesto por nidos de células epitelioides poligonales dispuestas en una red de vasos sanguíneos rodeados de colágeno. La fragilidad de la suplencia vascular lleva a cambios degenerativos secundarios con formación de áreas quísticas y cambios necróticos hemorrágicos. El patrón clásico histológico ocurre cuando las células tumorales forman configuraciones papilares compuestas de un tallo fibrovascular rodeado de varias capas de células epiteliales que contienen pequeños vasos sanguíneos formando así pseudopapilas. Este tumor tiene marcadores positivos para vimentina, enolasa especifica neuronal, alfa 1-antitripsina y alfa 1-antiquimotripsina. Es negativo para cromogranina, antígeno de membrana epitelial y citoqueratina $(1,13)$. Lo anterior puede explicar el origen endocrino del tumor siendo positivo para enolasa específica neuronal sin embargo la expresión de vimentina y alfa 1 antitripsina no apoya esta conclusión (6). 


\section{Características Imagenológicas}

Las características imagenológicas del tumor de Frantz reflejan los hallazgos patológicos del tumor como sus componentes sólidos y quísticos, hemorragia intratumoral, cápsula fibrosa, y en algunas ocasiones calcificaciones. Estas características hacen posible diferenciar a este tumor de otros tumores pancreáticos (1).

En ultrasonografía, el tumor se identifica como una masa grande circunscrita, bien demarcada con una capsula fibrosa de alta ecogenicidad la cual comprime diferentes estructuras sin invasión de ellas en la mayoría de los casos. En su arquitectura interna estos tumores usualmente tienen componentes quísticos y sólidos, los tumores más pequeños tienen mayor componente sólido y los tumores grandes mayor componente quístico demostrando reforzamiento acústico posterior. Algunos tumores se encuentran con áreas de baja ecogenicidad asociadas con áreas quísticas que sugieren necrosis hemorrágica. En ocasiones este tumor puede mostrar una alta ecogenicidad con reforzamiento acústico posterior indicando una masiva necrosis hemorrágica $(1,4,13,14)$.

La tomografía computarizada representa la mejor herramienta diagnostica en la evaluación de lesiones quísticas del páncreas. En el diagnóstico del tumor de Frantz se observa una lesión encapsulada de contornos definidos con una variedad de componentes sólidos y quísticos. Las porciones solidas son isodensas con respecto al parénquima pancreático. La porción quística puede variar de grado de atenuación según la extensión de necrosis hemorrágica contenida en el quiste. Se han reportado coeficientes de atenuación de 20 a 50 Unidades Hounsfield (ligeramente más alto que el contenido de la vesícula biliar) debido a productos sanguíneos $y$ detritus celulares vistos en aproximadamente un $20 \%$ en estos tumores. Adicionalmente este componente quístico varía en tamaño correspondiendo a la extensión de necrosis, coágulos sanguíneos y tejido necrótico tumoral. Sin embargo, esto no se correlaciona con el tamaño del tumor. Se debe considerar como primer diagnóstico diferencial el tumor de Frantz, a una gran masa en la cabeza o cola del páncreas de contornos definidos con porciones sólidas y quísticas, sin septos internos con rara presencia de metástasis en mujeres jóvenes $(1,2,15,16)$.

Sin embargo, cuando se compara la tomografía con la resonancia magnética, esta última permite diferenciar mejor la cápsula del tumor y la extensión de necrosis hemorrágica debido a su capacidad superior de resolución y contraste $(5,17,18)$. En imágenes potenciadas en T1 se aprecia una capsula fibrosa hipointensa con hemorragia interna de alta intensidad de señal la cual es una presentación característica de este tumor. Las porciones solidas son isointensas con respecto al parénquima pancreático o de baja intensidad de señal. En algunas ocasiones, se observan algunos focos de hiperintensidad dentro del tumor que corresponden a áreas de necrosis hemorrágica o detritus celulares $(5,11,16,19)$. En imágenes potenciadas en T2 se aprecia una intensidad de señal heterogénea (4). Con la administración de medio de contraste se evidencia un realce periférico heterogéneo en fase arterial temprana y un posterior llenado heterogéneo de la lesión en fase portal. Este tipo de realce ayuda a distinguir el tumor de Frantz de otros tumores pancreáticos como el tumor de los islotes pancreáticos que tiene un típico realce mayor que el páncreas (5).

En angiografía el tumor de Frantz es hipo o avascular y desplaza los vasos sanguíneos. Característica importante en el diagnóstico diferencial con el tumor de islotes pancreáticos $(1,4,20,21)$.

\section{Diagnóstico Diferencial.}

La apariencia en imagen del tumor sólido pseudopapilar del páncreas o tumor de Frantz puede superponerse con otros tumores pancreáticos como el adenoma seroso microquístico, neoplasia quística mucinosa, tumor quístico de los islotes pancreáticos, pancreatoblastoma y con patologías benignas como el pseudoquiste pancreático calcificado. Sin embargo, estos tumores raramente ocurren en pacientes menores de 30 años $(1,17,22)$. En imágenes de resonancia magnética, el adenoma microquístico seroso muestra una baja y heterogénea intensidad de señal en secuencias potenciadas en $\mathrm{T} 1$ con algunos focos de hemorragia hiperintensos similares al tumor de Frantz. Sin embargo, en secuencias potenciadas en T2 el adenoma microquístico seroso demuestra una cicatriz central de baja intensidad de señal que lo diferencia del tumor sólido pseudopapilar. La neoplasia quística mucinosa al igual que el tumor de Frantz son predominantemente quísticos con niveles líquido-líquido sugiriendo un componente hemorrágico. Sin embargo, el primero contiene mucina la cual puede ser hiperintensa en las secuencias potenciadas en T1 y T2. Además, no presenta realce capsular periférico como el tumor de Frantz $(17,23)$. Los tumores de los islotes pancreáticos ocurren en pacientes mayores y no son 
comunes en el sexo femenino como lo es el tumor sólido pseudopapilar. Otra característica que lo ayuda a diferenciarlo del tumor de Frantz es la alta vascularización y por consiguiente un mayor realce con el medio de contraste (24). El pancreatoblastoma es más agresivo que el tumor de Frantz y se diagnostica generalmente con metástasis al diagnóstico (25). Los pseudoquistes pancreáticos pueden semejar un tumor sólido pseudopapilar ya que pueden estar calcificados en su periferia como resultado de hemorragia interna. Sin embargo, el pseudoquiste pancreático se acompaña de clínica de pancreatitis y no presenta componentes sólidos (26).

\section{Pronóstico y tratamiento.}

El tumor de Frantz es un tumor de crecimiento lento de curso clínico benigno, pero con el potencial de adquirir comportamiento agresivo. Por lo tanto, la mejor opción de tratamiento es la resección completa del tumor. Si el tumor está limitado exclusivamente al páncreas el pronóstico es excelente con más del $95 \%$ de curación con la resección total $(1,9,27,28)$. En raras ocasiones este tumor presenta indicadores de potencial de malignidad como invasión venosa tumoral, alto grado de proliferación nuclear y nidos necrobióticos prominentes además de invasión capsular (29.30). Los reportes de metástasis son raros. El sitio de metástasis más común es el hígado (29.31). Aún si la resección completa del tumor o de las metástasis no es posible, las pacientes se benefician de cirugía de reducción del volumen tumoral $(9,12,27,29,32,33)$.

\section{Caso Clínico No. 1}

Paciente de sexo femenino de 14 años de edad, ingresa al servicio de urgencias del hospital militar central con dolor abdominal difuso de larga data el cual se acentuó en los últimos 2 días. Al examen físico, el abdomen se encontraba blando, con ruidos intestinales positivos y sensación de masa en epigastrio a la palpación profunda. Al interrogatorio no presenta antecedentes de importancia.

Con estos hallazgos se decide realizar tomografía abdominal con contraste la cual reporta masa que ensancha notoriamente la cabeza del páncreas con densidad heterogénea y con paredes gruesas de aproximadamente $5 \mathrm{~mm}$ de espesor, diámetro anteroposterior y transverso de $62.76 \mathrm{~mm}$ y $70.60 \mathrm{~mm}$ respectivamente. El cuerpo y la cola no mostraron alteraciones. El resto de órganos intraabdominales y estructuras vasculares se encontraron dentro de los límites normales. (Figura. 1)

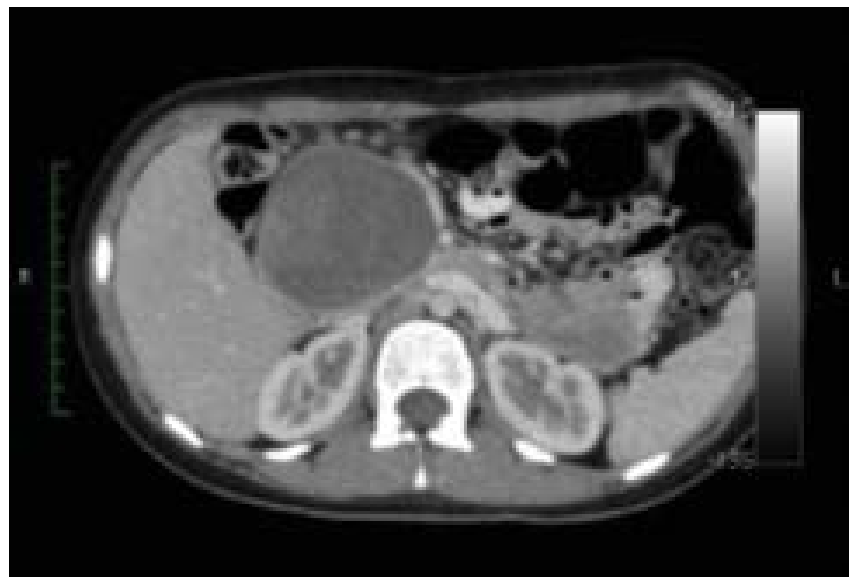

Figura 1. Corte axial de tomografía de abdomen contrastada con evidencia de lesión circular bien definida y encapsulada en la cabeza de páncreas la cual es de densidad heterogénea en su interior indicando hemorragia. Imagen realizada en el Hospital Militar Central de Bogotá.

El diagnóstico por tomografía es el de lesión de baja densidad que compromete la cabeza del páncreas en relación con tumor sólido pseudopapilar de la cabeza de páncreas. La paciente es llevada a cirugía donde se realiza resección total de la lesión. Posteriormente se traslada a la unidad de cuidados intensivos pediátricos presentando evolución satisfactoria. La muestra quirúrgica es analizada en el departamento de patología del Hospital Militar Central que reporta lesión redondeada lisa y brillante con abundante material hemorrágico y áreas de necrosis de aspecto carnoso y pardo rojizo. La histología reporta células en distribución pseudopapilar dando un diagnóstico definitivo de neoplasia solida pseudopapilar de la cabeza del páncreas. (Figura. 2)

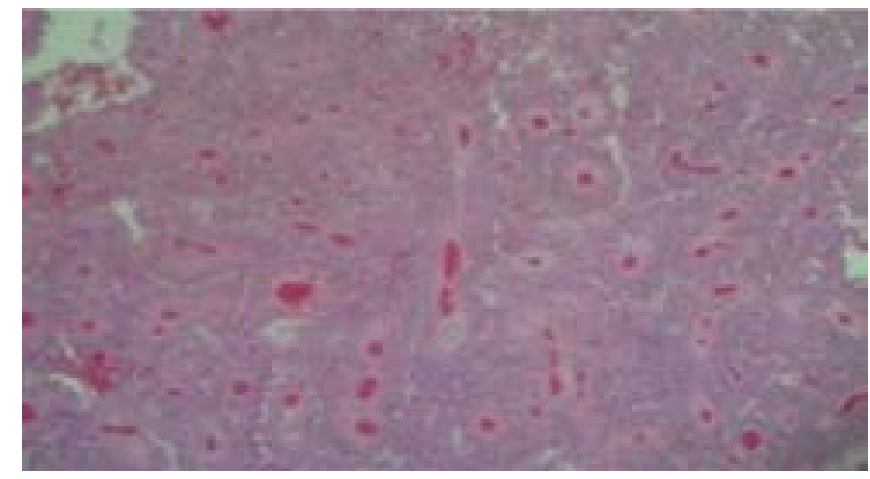

Figura 2. Descripción microscópica de la lesión en la cabeza del páncreas con presencia de células con proliferación en pseudopapilas. Imagen realizada en el departamento de patología del Hospital Militar Central de Bogotá. 


\section{Caso Clínico No. 2}

Paciente de sexo femenino de 19 años de edad, quien consulta por presentar dolor abdominal de varios meses de evolución asociado a hiporexia. Además, nota crecimiento gradual del perímetro abdominal. Niega pérdida de peso y no refiere ninguna otra sintomatología. No presenta antecedentes de importancia.

$\mathrm{Al}$ examen físico se encontró paciente en buen estado general con sensación de masa en mesogastrio.

Se realiza tomografía abdominal con contraste donde se evidencia masa originada en el cuerpo del páncreas con capsula hiperdensa y centro de densidad heterogénea de predominio hipodenso secundario a áreas de necrosis con diámetros anteroposterior y transverso de $220.6 \mathrm{~mm}$ x $270.1 \mathrm{~mm}$. (Figura. 3)

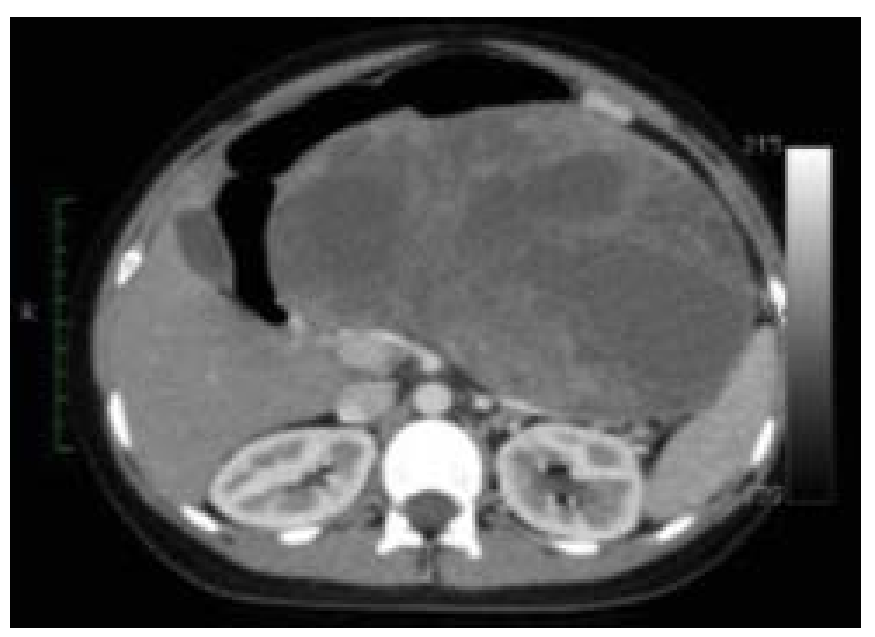

Figura 3. Corte axial de tomografía de abdomen contrastada con evidencia de lesión encapsulada de gran tamaño localizada en el cuerpo del páncreas que muestra áreas de mayor densidad en su interior en relación a hemorragia y áreas de menor densidad correspondientes a necrosis. Imagen realizada en el Hospital Militar Central de Bogotá.

El diagnóstico por tomografía es de una lesión quística dependiente de la cola del páncreas con áreas de necrosis en su centro con un diagnóstico diferencial de tumor no funcionante de los islotes pancreáticos y tumor solido pseudopapilar. La paciente se programa para cirugía, se realiza resección total de la masa y es trasladada a la unidad de cuidados intensivos donde evoluciona satisfactoriamente. La muestra quirúrgica es llevada al departamento de patología del Hospital
Militar Central que reporta lesión tumoral encapsulada con proliferación de pseudopapilas, abundantes capilares de tamaño variable. Células neoplásicas sueltas y áreas de necrosis con diagnostico final de tumor solido pseudopapilar de la cola del páncreas. (Figura. 4)

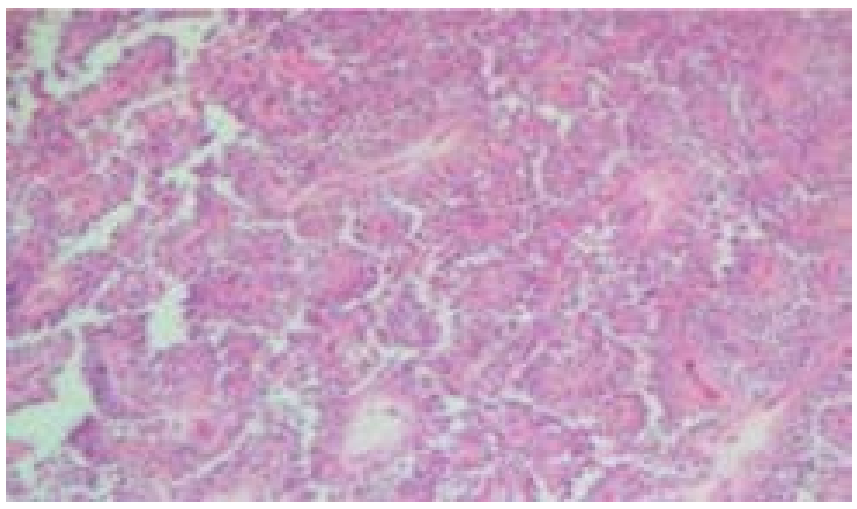

Figura 4. Descripción microscópica de la lesión de la cola del páncreas que evidencia lesión encapsulada con proliferación de pseudopapilas y áreas de necrosis. Imagen realizada en el departamento de patología del Hospital Militar Central de Bogotá.

\section{Discusión}

El tumor de Frantz o tumor sólido pseudopapilar del páncreas es una lesión rara que ocurre principalmente entre la segunda y cuarta década de la vida. Es de bajo potencial maligno y se caracteriza por tener un pronóstico favorable (34). No tiene un origen celular muy claro y fue descrito por primera vez por la doctora Virginia Frantz en 1959 como un tumor papilar del páncreas benigno o maligno donde reportó 3 casos (35). Posteriormente recibe la primera caracterización patológica en 1970 por Hamoudi et al, reportando un caso de una niña de 12 años de edad (36). En 1981 Kloppel et al, reportan 5 casos y concluye que esta lesión tenia características propias y que debería ser clasificado como una variedad diferente de tumor del páncreas exocrino (37). Sin embargo, la nomenclatura de esta entidad ha venido cambiando a través del tiempo y permanecía confusa para algunos autores. Esta entidad ha sido llamada tumor sólido y papilar, tumor solido-quístico, tumor quístico papilar, neoplasia epitelial papilar, neoplasia sólida y papilar, neoplasia solida epitelial y pseudopapilar, neoplasia sólida y quística de células acinares y tumor de Frantz. En muchas ocasiones esta neoplasia fue mal diagnosticada como tumor de los islotes pancreáticos no funcionante, adenocarcinoma, cistoadenoma o pseudoquiste. Finalmente, en 1996, la asociación mundial de la salud reconoce el tumor y lo 
clasifica como "tumor sólido pseudopapilar" por la clasificación histológica internacional del páncreas exocrino. Actualmente este tumor ha sido reconocido por sus características únicas de neoplasia solida pseudopapilar y se han documentado hasta el momento más de 700 casos en la literatura $(2,3,6,34,38,39)$.

\section{Conclusión}

El tumor de Frantz o tumor solido pseudopapilar del páncreas, es una neoplasia que se presenta principalmente en niñas adolescentes y en mujeres jóvenes. Este tumor ha sido reconocido por la organización mundial de la salud por sus características únicas de neoplasia sólida pseudopapilar. Es de bajo potencial maligno y se caracteriza por tener un pronóstico favorable. Como se presentó en esta revisión, la mayoría de las pacientes presentan síntomas y hallazgos al examen físico inespecíficos, y en algunas ocasiones es un diagnóstico incidental al realizar estudios imagenológicos y cirugías abdominales por otras razones. Los hallazgos por imagen son mejor valorados con la tomografía computarizada y con la resonancia magnética que en la mayoría de los casos le dan una caracterización definitiva que hace posible diferenciarlo de otros tumores y lesiones pancreáticas benignas. Por lo tanto, el papel del radiólogo es de vital importancia ya que con una adecuada caracterización imagenológica del tumor nos va a ayudar a realizar un diagnóstico y contribuir a un tratamiento oportuno en estas pacientes.

\section{Conflicto de intereses}

Los autores declaran no tener de manera directa o indirecta ningún tipo de conflicto de intereses financieros, académicos o laborales que puedan poner en peligro la validez de este estudio.

\section{Financiación}

Este trabajo se realizó con recursos propios.

\section{Referencias}

1. Chung E, Travis M, Conran R. Pancreatic tumors in children: Radiologic-Pathologic Correlation. Radiographics. 2006;26:1211-1238.

2. Baek JH, Lee JM, Kim SH, Kim SJ, Kim S, Lee JY et al. Small $(<3 \mathrm{~cm})$ Solid Pseudopapillary Tumors of the Pancreas at Multiphasic Multidetector CT. Radiology. 2010;(1):97-106.

3. Escobar MA, Bond BJ, Schopp J. Solid Pseudopapillary Tumour (Frantz's Tumour) of the Pancreas in Childhood.
BMJ Case rep. 2014;doi:10.1136/bcr-2013-200889

4. Procacci C, Graziani R, Bicego E. Papillary cystic neoplasm of the páncreas: radiological findings. Abdom Imaging 1995;20(6):554-558.

5. Cantisani V, koenraad M, Levy A, Glickman J, Ricci P, Passariello $\mathrm{R}$ et al. MR imaging features of Solid Pseudopapillary Tumor of the Pancreas in Adult and Pediatric Patients. AJR. 2003;181:395-401

6. Coleman K, Doherty M, Bigler S. Solid-Pseudopapillary Tumor of the Pancreas. Radiographics. 2003;23:1644-1648.

7. Morales A, Ruiz J, Esteves H, Robles G, Díaz V. Papillary-cystic neoplasm of the pancreas: a sex steroid dependent tumor. Int J Pancreatol. 1998;24:219-225

8. Von Herbay A, Sieg B, Otto H. Solid-CysticTumor of the Pancreas: an endocrine neoplasm?. Virchows Arch A Pathol Anat Histopathol. 1990;416:535-538.

9. Mao C, Guvendi M, Domenico D, Kim K, Thomford, Howard J. Papillary Cystic and Solid Tumors of the Pancreas: a pancreatic embryonic tumor? Studies of three cases and a cumulative review of world's experience. Surgery 1995; 118:821-828

10. Massaro M, Estrada T, Mejia P. Evaluación Imagenológica de las Lesiones Quísticas del Páncreas. Rev Colomb Radiol. 2010;21(2):2882-2900.

11. Choi J, Kim M, Kim J, Kim S, Lim J, Oh j et al. Solid Pseudopapillary Tumor of the Pancreas: Typical and Atypical Manifestations. AJR. 2006;187:178-186.

12. Sclafani L, Reuter V, Coit D, Brennan M. The Malignant Nature of Papillary and Cystic Neoplasm of the Pancreas. Cancer 1991;68(1):153-158

13. Kloppel G, Morohoshi T, John HD. Solid and Cystic acinar cell tumor of the pancreas: a tumor un young women with favorable prognosis. Virchows Arch A Pathol Anat Histol. 1981;392(2):171-183.

14. Lee D, Yi B, Lim J, Ko Y. Sonographic findings of solid and papillary epithelial neoplasm of the pancreas. J Ultrasound Med. 2001;20:1229-1232.

15. Byung I, Ki K, Man H, Yong K, Chu-wan K. Solid and Papillary Epitelial Neoplasms of the Pancreas: CT Findings. Radiology 1988;166:413-416

16. Sun C, Lee W, Choi J, Oh J, Choi S. Solid Pseudo-papillary tumors of the pancreas: 14 years of experience. ANZ J Surg. 2005;75(8):684-689

17. Mergo P, Helmberger T, Buetow P, Helmberger R, Ros P. Pancreatic Neoplasms: MR Imaging and Pathologic Correlation. Radiographics 1997;17:281-301.

18. Morohoshi T, Held G, Kloppel G. Exocrine pancreatic tumors and their histological classification: a study based on 167 autopsy and 97 surgical cases. Histopathology 1983;7:645-661

19. Ohtomo K, Furui S, Onoue M et al. Solid and papillary ep- 
ithelial neoplasm of the pancreas: MR imaging and pathologic correlation. Radiology 1992;184(2):567-570.

20. Friedman AC, Lichtenstein J, Fishman E, Oertel J, Dachman A, Siegelman S. Solid and Papillary Epithelial Neoplasm of the pancreas. Radiology 1985;154(2):333337

21. Buetow P, Buck J, Pantongrag-Brown L, Beck K, Ros P, Adair C. Solid and Papillary Epithelial Neoplasm of the Pancreas: Imaging-pathologic correlation in 56 cases. Radiology. 1996;199:707-711

22. Takeshita K, Furui S, Makita K, et al. Cystic cell islet tumors: radiologic findings in three cases. Abdom Imaging 1994;19:225-228.

23. Minami M, Itai $Y$, Ohtomo K. Cystic endoplasms of the pancreas: comparison of MR imaging with CT. Radiology 1989;171:53-56.

24. Kelekis NL, Semelka RC, Molina PL, Doerr ME. ACTH-secreting islet cell tumor: appearances on dynamic gadolinium-enhanced MRI. Magn Reson Imaging 1995; 13:641-644.

25. Klimstra DS, Wring B, Adair CF, Heffes CS. Pancreatoblastoma: a clinicopathologic study and review of the literature. Am J Surg Pathol 1995;16:371-389.

26. Kehagias D, Smyrniotis V, Gouliamos A, Vlahos L. Cystic pancreatic neoplasms. Int J Pancreatol 2000;28:223-230.

27. Solcia E, Capella C, Kloppel G. Atlas of tumor Pathology: tumors of the pancreas. Washington DC: Armed Forces Institute of Pathology, 1997.

28. Lam KY, Lo CY, Fan ST. Pancreatic solid-cystic-papillary tumor: clinicopathologic features in eight patients from Hong Kong and review of the literature. World J Surg 1999;23:1045-1050.

29. Nishihara K, Nagoshi M, Tsuneyoshi M, Yamagushi K, Hayashi I. Papillary cystic tumors of the pancreas: assessment of their malignant potential. Cancer 1993;71:82-92.
30. Shimizu M, Matsumoto T, Hirokawa M, et al. Solid-pseudopapillary carcinoma of the pancreas: a case report. Pathol Int 1999;49:231-234.

31. Matsunou H, Konishi F. Papillary-cystic neoplasm of the pancreas: a clinicopathologic study concerning the tumor aging and malignancy of nine cases. Cancer 1990;65(2):283-291

32. Papavramidis T, Papavramidis S. Solid pseudopapillary tumors of the pancreas: review of 718 patients reported in English literature. J Am Coll Surg 2005;200(6):965-972

33. Wang KS, Albanese C, Dada F, Skarsgard ED. Papillary cystic neoplasm of the pancreas: a report of three pediatric cases and literature review. J Pediatr Surg 1998;33(6):842-845

34. Choi J, Kim M, Kim J, Kim S, Lim J, Oh j et al. Solid Pseudopapillary Tumor of the Pancreas: Typical and Atypical Manifestations. AJR. 2006;187:178-186.

35. Frantz V. Tumors of the pancreas. In: Atlas of tumor pathology. Section 7, fascicles 27 and 28. Washington DC: Armed Forces Institute of Pathology. 1959;32-33.

36. Hamoudi A, Misugi K, Grosfeld J, Reiner C. Papillary endothelial neoplasm of pancreas in a child: report of a case with electron microscopy. Cancer 1970;26(5):11261134.

37. Kloppel G, Morohoshi T, John HD. Solid and Cystic acinar cell tumor of the pancreas: a tumor un young women with favorable prognosis. Virchows Arch A Pathol Anat Histol. 1981;392(2): 171-183.

38. Hamilton S, Aaltonen L. WHO classification of tumors: pathology and genetics of tumors of the digestive system. Lyon, France: IARC Press. 1996;220.

39. Zhou H, Cheng W, Lam KY, Chan GC, Khong PL, Tam PK. Solid-Cystic papillary tumor of the pancreas in children. Pediatr Surg Int 2001;17(8):614-620 